\title{
Effects of Dimethyl Sulfoxide on the Globally Ischemic Heart: Possible General Relevance to Hypothermic Organ Preservation ${ }^{1}$
}

\author{
MARSHAL SHLAFER, ${ }^{2}$ PAULA F. KANE, AND MARVIN M. KIRSH \\ Departments of Pharmacology and Surgery, Section of Thoracic Surgery, The University of Michigan \\ Medical School, Ann Arbor, Michigan 48109
}

Dimethyl sulfoxide (DMSO) is routinely used to prevent freeze-thaw-induced damage to a variety of biological systems. In that context, relatively high drug concentrations in excess of one molar are usually used. However, lower concentrations of the drug produce many pharmacological effects (26) which may be efficacious for reducing hypoxia- or ischemia-induced cell damage which is often encountered during organ preservation protocols that do not necessarily involve freezing.

During the course of a study of several interventions which might be useful to prevent damage to the globally ischemic heart, we observed a relatively discrete but perhaps important action of dimethyl sulfoxide. When added to hypothermic cardioplegic solutions, $70 \mathrm{~m} M$ dimethyl sulfoxide significantly and consistently prevented alterations of mitochondrial oxidative phosphorylating activity after $2 \mathrm{hr}$ of global ischemia and reperfusion. Based on these findings, the known pharmacological spectrum of action of dimethyl sulfoxide, and a postulated mechanism by which it exerted its protective effects, we were led to speculate that the drug might have more widespread applicability to organ preservation than it currently enjoys.

Received June 17, 1981; accepted September 8, 1981.

${ }^{1}$ This work was supported in part by a Grant-in-Aid from the Michigan Heart Association.

${ }^{2}$ Send correspondence and reprint requests to: Dr. Marshal Shlafer, Department of Pharmacology, University of Michigan Medical School, M-6322 Medical Sciences Bldg. \# 1, Ann Arbor, Mich. 48109.

\section{MATERIALS AND METHODS}

\section{Model of Cardiac Global Ischemia}

We used hearts isolated from healthy New Zealand rabbits weighing approximately $1 \mathrm{~kg}$. The rabbit was stunned by a blow to the head, exsanguinated, and the thorax was opened. The heart and lungs were quickly removed and the ascending aorta was cannulated with a blunted 12gauge needle. The cannula was quickly mounted on a nonrecirculating nonpulsatile perfusion circuit, and retrograde aortic perfusion with an oxygenated normothermic $\left(37^{\circ} \mathrm{C}\right)$ physiological saline solution (PSS; see below) was begun. A polyethylene catheter was tied into the pulmonary artery for collecting and measuring coronary effluent and lactate dehydrogenase (LDH) released into it. The lungs were removed once perfusion was established, and the remaining pulmonary vessels were ligated. A saline-filled, balloon-tipped catheter connected to a Statham pressure transducer was inserted through a left atriotomy into the left ventricular chamber to measure left ventricular developed pressure (LVDP) and its first derivative, $\mathrm{LV} d P / d t$. A saline-filled calibrated syringe connected by stopcock to the catheter was used to vary the volume of the intraventricular balloon. A thermostated water jacket was raised around the heart. The hearts were allowed to beat spontaneously, and during both preischemic and postischemic perfusion with oxygenated PSS perfusion pressure was maintained at $75 \mathrm{~mm} \mathrm{Hg}$ by adjusting the speed of the perfusion pump. We 
recorded LVDP, LV $d P / d t$, perfusion pressure, and the isolated heart electrogram on an oscillograph throughout the experiment. All hearts received an initial 30-min equilibration perfusion with oxygenated PSS at $37^{\circ} \mathrm{C}$, and then preischemic functional indicators described below were recorded. Hearts were then assigned randomly to one of the experimental or control (nonischemic) groups.

Most hearts were made globally ischemic. After equilibration they were perfused for $5 \mathrm{~min}$ with one of two hypoxic (nonoxygenated) substrate-free hypothermic $\left(20\right.$ or $\left.27^{\circ} \mathrm{C}\right)$ cardioplegic test solutions, then made ischemic by terminating perfusion. The thermostated jacket surrounding the heart was filled with the same test solution, at the same temperature, as that which had been perfused. This was done to prevent desiccation and to maintain temperature during the long perfusion-free period. Hypothermic temperatures were regulated with a Haake refrigerated circulator. At the end of a 2-hr uninterrupted period of global ischemia the jacket was drained and a second 5-min perfusion with the identical test solution was begun. The hearts were then reperfused for $60 \mathrm{~min}$ with the normothermic oxygenated PSS as was used during preischemic equilibration. The brief postischemic reperfusion with cardioplegic solution was predicated upon the assumption that it would be preferable to wash out any toxic metabolites which might have accumulated in the extracellular spaces during ischemia, and before providing the heart with a more physiological, oxygenated solution which was conducive to contraction. Additionally, in some intraoperative settings the heart is briefly reperfused with cardioplegic solution before reperfusing it with blood from the cardiopulmonary bypass circuit after ischemia, primarily for the reason noted above.

Postischemic recovery of function was considered to be that which we measured at the end of the entire $65-\mathrm{min}$ reperfusion pe- riod ( $5 \mathrm{~min}$ with cardioplegic solution and 60 min with oxygenated PSS).

Five hearts were not made ischemic, but instead were perfused for $220 \mathrm{~min}$ with oxygenated PSS at $37^{\circ} \mathrm{C}$. Function was measured after the 30 -min equilibration period and again $190 \mathrm{~min}$ later. This last measurement coincided with the time at which postischemic function was measured in hearts which had been made ischemic. The nonischemic hearts therefore served as controls.

The major indicators of cardiac status which we measured were spontaneous heart rate, coronary flow (which was inversely proportional to coronary vascular resistance, since flow always was measured at the same perfusion pressure, $75 \mathrm{~mm} \mathrm{Hg}$ ), LDH release in the pulmonary artery effluent, and LVDP and $d P / d t$. These latter measurements of ventricular contractility were assembled as LV function (Starling) curves: LVDP was measured at various LV end-diastolic pressures which were, in turn, a function of $\mathrm{LV}$ volume. $\mathrm{LV}$ volume was varied experimentally by incrementally increasing the volume of the saline-filled intraventricular balloon (33). The function curves were constructed after the $30-\mathrm{min}$ equilibration period and again after $65 \mathrm{~min}$ of reperfusion. At all other times, balloon volumes were adjusted to give an enddiastolic pressure of approximately $5 \mathrm{~mm} \mathrm{Hg}$.

\section{Mitochondria}

Once postreperfusion functional measurements were made the heart was removed from the perfusion circuit and mitochondria were isolated from left ventricular and septal myocardium (32) by differential centrifugation, in a medium containing $\mathrm{KCl}$ $(0.18 M)$, Tris-EDTA (10 mM, pH 7.40, at $\left.4^{\circ} \mathrm{C}\right)$ and bovine serum albumin $(0.5 \%$, $\mathrm{w} / \mathrm{v})$. The respiratory and phosphorylating activity of these organelles were evaluated polarographically (Gilson Oxygraph equipped with a Clark-type electrode). Details of the isolation protocol are described 
elsewhere (32). For convenience the composition of the media used for assaying mitochondrial function, and details of the assay conditions, are given in Table 2 , in which the pertinent experimental data are summarized.

In some experiments we tested the direct effects of dimethyl sulfoxide on the function of mitochondria isolated from nonischemic-nonperfused rabbit hearts. For these experiments we used a vibrating platinum electrode, since preliminary studies showed that high dimethyl sulfoxide concentrations ( $0.42 M$ or more) caused the Clark electrode to respond erratically, even when mitochondria were not present in the assay cuvette. Presumably this was due to an effect of the drug on the permeability or electrical properties of the Clark electrode's Teflon membrane.

\section{Perfusates and Chemicals}

The composition of the standard oxygenated perfusate (PSS) was $(\mathrm{m} M): \mathrm{NaCl}, 118$; $\mathrm{KCl}$, 5.4; $\mathrm{MgCl}_{2}, 1.2 ; \mathrm{NaHCO}_{3}, 25$; $\mathrm{KH}_{2} \mathrm{PO}_{4}, 1 ; \mathrm{CaCl}_{2}, 2.4$; and D-glucose, 10. The solution had a $\mathrm{pH}$ of 7.40 after equilibration with a mixture of $95 \% \mathrm{O}_{2}$ and $5 \%$ $\mathrm{CO}_{2}$. All hearts were perfused with this solution during the 30 -min preischemic equilibration and again during the last $60 \mathrm{~min}$ of postischemic reperfusion; nonischemic controls were perfused exclusively with this solution.

The test solution perfused briefly before and after ischemia was a procaine-free modification of the St. Thomas' Hospital Solution described by Jynge and colleagues (17). It contained $(\mathrm{mM}): \mathrm{NaCl}, 92 ; \mathrm{KCl}, 15$; $\mathrm{MgCl}_{2}, 15 ; \mathrm{NaHCO}_{3}, 25 ; \mathrm{KH}_{2} \mathrm{PO}_{4}, 1$; and $\mathrm{CaCl}_{2}, 1.2$. It was used in the hypoxic state and had a $\mathrm{pH}$ of 7.70 .

Solutions containing dimethyl sulfoxide (experimental drug grade; Crown Zellerbach Corporation, Camas, Wash.) were made by replacing appropriate volumes of water with pure drug (28).

\section{RESULTS}

Figure 1 shows left ventricular developed pressures, measured at various enddiastolic pressures, for hearts in the nonischemic control and ischemic-reperfused groups. The data show that $2 \mathrm{hr}$ of global ischemia plus reperfusion caused the expected decreases of postischemic contractile function, compared to function measured before ischemia or in the nonischemic controls; that cardioplegia at $20^{\circ} \mathrm{C}$ provided significantly better preservation of pressure development than at $27^{\circ} \mathrm{C}$; and that supplementing cardioplegia solution with 70 $\mathrm{m} M$ dimethyl sulfoxide produced no additional effects, beneficial or deleterious, compared to the appropriate drug-free solution. Measurements of maximum rates of left ventricular pressure development

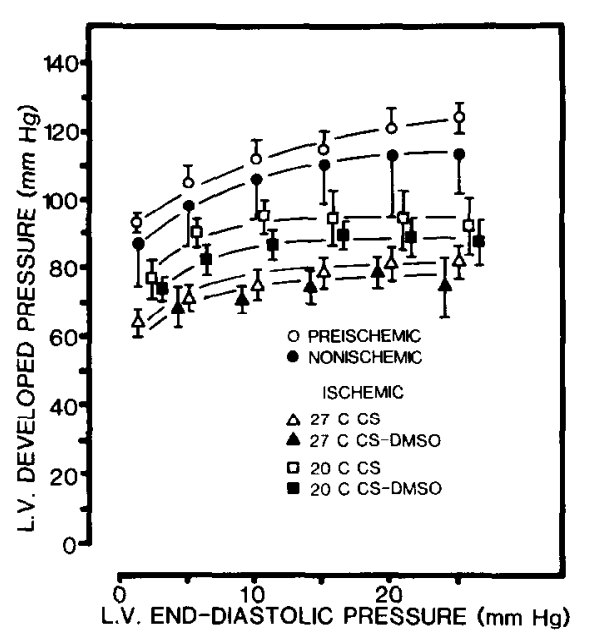

FIG. 1. Left ventricular developed pressure is plotted as a function of left ventricular end-diastolic pressure, which was adjusted by varying the volume of a left intraventricular balloon. Prolonged perfusion of nonischemic hearts resulted in a slight decrease of left ventricular function, compared to values measured before ischemia (preischemic values). Maximum left ventricular developed pressure after $2 \mathrm{hr}$ global ischemia and $1 \mathrm{hr}$ was significantly greater in hearts made ischemic at 20 than at $27^{\circ} \mathrm{C}$. For a given temperature during ischemia, however, adding $70 \mathrm{mM}$ dimethyl sulfoxide (DMSO) to the cardioplegic solution (CS) had no consistent or significant effect on postischemic contractile function. 
$(d P / d t$, Table 1) or left ventricular compliance (left ventricular end-diastolic pressure as a function of end-diastolic volume) showed the same between-group relationships (data not presented).

Table 1 summarizes values for heart rate, coronary flow, lactate dehydrogenase release, and tissue $\mathrm{Ca}$ content. The major pertinent observation was that supplementing CS with $70 \mathrm{~m} M$ dimethyl sulfoxide had no significant effect on any of these variables.

Table 2 summarizes the in vitro respiratory activity of mitochondria isolated from hearts in the various groups. Glutamate plus malate were the respiratory substrates. There are three major effects seen in these data. First, perfusing hearts for $3.5 \mathrm{hr}$ with a normothermic, colloid-free solution markedly reduces State 3 (ADP-stimulated) respiratory rates and the respiratory control ratio ( $R C R$ ) and oxidative phosphorylation rate (OPR), two functional indicators which are based, in part, on the State 3 respiratory rate. This effect is seen by examining values reported for nonperfused heart mitochondria. Next, the data show that perfusing hearts with $\mathrm{CS}$ at either 27 or $20^{\circ} \mathrm{C}$, and making them globally ischemic at those temperatures, results in further and generally significant decreases of State 3 rates, RCR and OPR, compared to the appropriate control, namely those values for the nonischemic control group. Finally, and perhaps most important, the data show that supplementing hypothermic CS with $70 \mathrm{mM}$ dimethyl sulfoxide increased State 3 respiratory rates to values which were not significantly different from those of nonischemic control mitochondria, but were significantly greater than those measured in the appropriate drug-free CS-treated group. Increasing State 3 respiratory rates also increased RCR and OPR values. Based on analysis of variance, we found no betweengroup differences of mitochondrial ADP:O ratios; however, this functional indicator is relatively resistant to all but the most severe ischemic insults, and is considered to be a relatively insensitive index of $\mathrm{mi-}$ tochondrial integrity.

Table 3 summarizes the direct effects of 0.14 to $1.4 M(1$ to $10 \%, \mathrm{v} / \mathrm{v})$ dimethyl sulfoxide on mitochondrial respiratory control and oxidative phosphorylation rates. The only significant effect produced by the drug was at $1.4 \mathrm{M}$, manifest as a decrease of the respiratory control ratio, due to decreased State 3 respiratory rates.

\section{DISCUSSION}

Our results emanated from a larger study designed to improve upon myocardial preservation during induced intraoperative ischemia, afforded by the clinical standard, hypothermic cardioplegia. It was predicated upon growing evidence that reoxygenation of ischemic (and, therefore hypoxic or anoxic) organs causes the generation or excessive accumulation of cytotoxic oxygen metabolites (superoxide anion; hydroxyl radical; hydrogen peroxide), presumably due to depletion of cellular enzymes which normally and rapidly convert these metabolites to less toxic products. Mitochondria appear to be an important site for the generation of these metabolites $(2,8,20,21)$, some of which are free radicals, and so it is likely that one of the important targets of the cytotoxicity would be the mitochondria themselves.

In our study we found that the effects of $70 \mathrm{~m} M$ dimethyl sulfoxide were limited to improvements of mitochondrial oxidative phosphorylating activity. The drug did not significantly alter other indicators of cardiac function, the most important of which are ventricular pressure development and compliance. However, perhaps a more relevant observation is that although higher concentrations of the drug can be cardiotoxic $(11,28)$, and lower concentrations can exacerbate cell damage caused by pathological states (10), we observed no adverse effects attributable to the drug, based on 


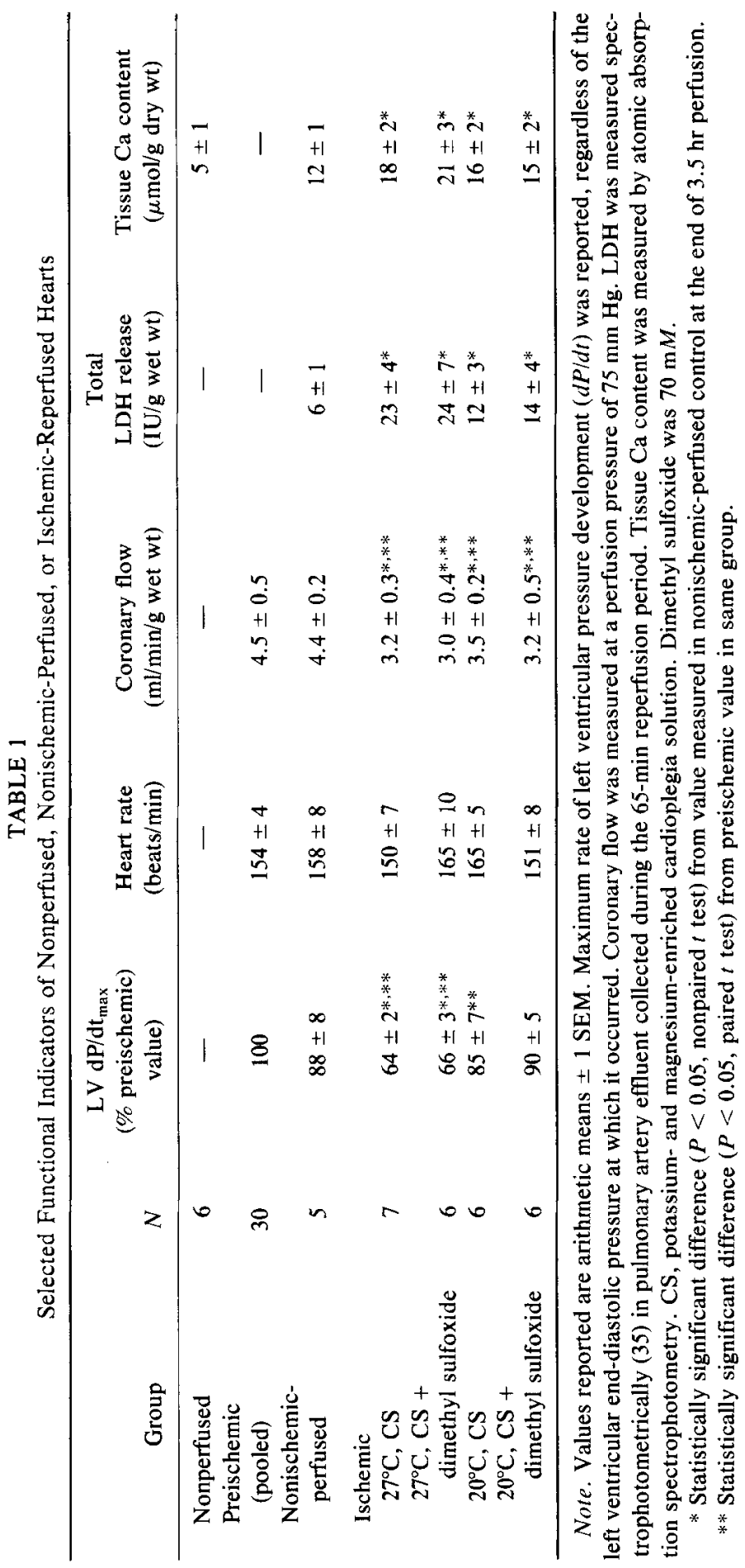


TABLE 2

Respiratory Function ${ }^{a}$ of Mitochondria Isolated from Nonischemic or Ischemic-Reperfused Hearts

\begin{tabular}{|c|c|c|c|c|c|c|}
\hline \multirow[b]{2}{*}{ Group } & \multirow[b]{2}{*}{$N$} & \multirow[b]{2}{*}{ State $3^{b}$} & \multirow[b]{2}{*}{ State $4^{b}$} & \multirow[b]{2}{*}{$\mathrm{RCR}^{b}$} & \multicolumn{2}{|c|}{ Respiratory rates } \\
\hline & & & & & ADP:O ratio & $\mathrm{OPR}^{b}$ \\
\hline $\begin{array}{l}\text { Nonperfused } \\
\text { Nonischemic }\end{array}$ & 6 & $174 \pm 32$ & $13 \pm 3$ & $14.0 \pm 0.7$ & $2.58 \pm 0.06$ & $448 \pm 89$ \\
\hline control & 5 & $109 \pm 14$ & $11 \pm 1$ & $9.9 \pm 0.5$ & $2.54 \pm 0.02$ & $271 \pm 36$ \\
\hline \multicolumn{7}{|l|}{ Ischemic } \\
\hline $\begin{array}{l}27^{\circ} \mathrm{C}, \mathrm{CS}^{c} \\
27^{\circ} \mathrm{C}, \mathrm{CS}+\end{array}$ & 7 & $68 \pm 7^{*}$ & $10 \pm 1$ & $6.7 \pm 0.4^{*}$ & $2.46 \pm 0.02$ & $165 \pm 15^{*}$ \\
\hline dimethyl sulfoxide & 6 & $104 \pm 12^{* * *}$ & $12 \pm 1$ & $8.5 \pm 0.4^{* *}$ & $2.63 \pm 0.09$ & $273 \pm 30^{-k *}$ \\
\hline $\begin{array}{l}20^{\circ} \mathrm{C}, \mathrm{CS} \\
20^{\circ} \mathrm{C}, \mathrm{CS}+\end{array}$ & 6 & $89 \pm 9$ & $10 \pm 1$ & $8.7 \pm 0.7$ & $2.47 \pm 0.06$ & $220 \pm 21$ \\
\hline dimethyl sulfoxide & 6 & $123 \pm 18^{*+k}$ & $16 \pm 2 *$ & $8.2 \pm 0.9$ & $2.40 \pm 0.12$ & $295 \pm 44^{* *}$ \\
\hline
\end{tabular}

${ }^{a}$ Values shown are arithmetic means \pm 1 SEM. Mitochondrial function was assayed in a medium containing $220 \mathrm{~m} M$ sucrose, $4.4 \mathrm{mM} \mathrm{K} \mathrm{KPO}_{4}, 7.5 \mathrm{~m} M$ Tris-glutamate, $7.5 \mathrm{~m} M$ Tris-malate, approximately $1 \mathrm{mg} / \mathrm{ml}$ mitochondrial protein, $500 \mathrm{nmol} \mathrm{Na}$-ADP, in $13 \mathrm{mM}$ morpholinopropanesulfonic acid buffer, pH 7.40 , at $30^{\circ} \mathrm{C}$. Total assay volume was $1.605 \mathrm{ml}$. A Gilson Oxygraph with Clark-type oxygen electrode was used.

${ }^{b}$ Respiratory rates are reported as natom $\mathrm{O} / \mathrm{min} / \mathrm{mg}$ protein. RCR, Respiratory control ratio (State 3 rate/ State 4 rate). OPR, Oxidative phosphorylation rate (State 3 rate $\times$ ADP:O ratio).

c CS, potassium- plus magnesium-enriched cardioplegia solution. Dimethyl sulfoxide was $70 \mathrm{~m} M$.

* Significantly different $(P<0.05)$ from nonischemic control value, based on nonpaired $t$ test.

** Significantly different $(P<0.05$ ) from comparable drug-free group, based on nonpaired $t$ test.

TABLE 3

Direct Effects of Dimethyl Sulfoxide on Respiratory Control Ratios and Oxidative Phosphorylation Rates of Normal Rabbit Heart Mitochondria ${ }^{a}$

\begin{tabular}{ccc}
\hline $\begin{array}{c}\text { Dimethyl } \\
\text { sulfoxide }(M)\end{array}$ & $\mathbf{R C R}^{b}$ & OPR $^{b}$ \\
\hline 0 & $100 \%$ & $100 \%$ \\
0.14 & $16 \pm 8$ & $3 \pm 5$ \\
0.42 & $-5 \pm 5$ & $4 \pm 6$ \\
0.84 & $-12 \pm 2$ & $4 \pm 4$ \\
1.41 & $-29 \pm 8^{*}$ & $-5 \pm 4$ \\
\hline
\end{tabular}

${ }^{a}$ Three to five preparations were studied. Mitochondria were isolated from nonperfused hearts. Control values and experimental conditions are noted in Table 2. Incubation medium was made by replacing appropriate volumes of water with drug-grade dimethyl sulfoxide.

${ }^{b}$ Values given are arithmetic means \pm SEM, and are expressed as a percentage of the value obtained in dimethyl sulfoxide-free medium (which was normalized to $100 \%$ ). RCR, Respiratory control ratio (State 3 rate of oxygen consumption/subsequent State 4 rate); OPR, oxidative phosphorylation rate (State 3 rate $\times$ accompanying ADP:O ratio).

* Significantly different $(P<0.05$, paired $t$ test) from drug-free control. the experimental conditions we imposed and on the measurements we made.

Dimethyl sulfoxide produces many pharmacological effects which are relevant to organ preservation in general (27), and to myocardial preservation in particular $(27$, 29). It can alter the activity of a variety of enzymes $(3,18,26,27,30)$, and can have direct effects on mitochondria $(5,6)$. However, the circumstantial evidence which best supports a putative mechanism for the protective action of dimethyl sulfoxide seems to center on its effects on "reoxygenation-mediated" damage (14).

Finney and colleagues (12) reported that dimethyl sulfoxide, combined with hydrogen peroxide, lessened myocardial infarct size in porcine hearts subjected to coronary artery ligation in situ. Leon and colleagues (19) also demonstrated that chronic dimethyl sulfoxide pretreatment lessened the severity of isoproterenol-induced myocardial necrosis and prevented ventricular aneurysm development. High-dose isopro- 
terenol treatment is thought to produce a relative myocardial ischemia due to druginduced increases of oxygen demand in great excess of oxygen supply. Both Finney and Leon speculated that dimethyl sulfoxide protected by increasing oxygen delivery to ischemic tissue, as has been reported to occur with normal tissues (23).

However, these observations do not appear to be mechanistically consistent with the growing evidence about reoxygenationmediated cell damage, or some of the experimental data concerning ways to reduce that component of damage. For example, gradual reoxygenation delays the onset of reoxygenation-mediated damage, and so enhanced cellular oxygen delivery, particularly in combination with hydrogen peroxide, one of the implicated toxic oxygen metabolites, would not be expected to lessen damage. Also, administration of mitochondrial poisons such as $\mathrm{CN}^{-}$, which inhibits mitochondrial electron transport and, presumably, mitochondrial oxygen metabolite generation, prevents the development of irreversible mitochondrial damage and other manifestations of reoxygenation damage (13) which lead to myocardial cell death. Therefore, an intervention which simply enhances cell oxygen tensions upon reperfusion would either enhance oxygen-mediated damage, hasten its onset, or both.

The role of free-radical formation and membrane lipid peroxidation by free radicals as a component of oxygen metaboliterelated pathology, is well established $(7,25$, 34). Of relevance to this study, then, are the observations that dimethyl sulfoxide is a free-radical scavenger. This property has been cited as a primary mechanism by which the drug protects against radiationinduced damage (1), prevents the diabetogenic effects of alloxan (15), inhibits some hepatic microsomal enzymes (4), and inhibits prostaglandin biosynthesis $(9,16$, 23). Inhibition of prostaglandin biosynthesis and scavenging of free radicals prob- ably explain the drug's ability to inhibit platelet aggregation and inflammation. Therefore, we speculate that since mitochondria generate free radicals, and since dimethyl sulfoxide can scavenge these, this constitutes a reasonable mechanism for its discrete but significant effects. To support this, we have found ( 30 ) that superoxide dismutase and catalase, enzymes known to convert cytotoxic oxygen metabolites to less toxic products, also enhance the protective effects of cold cardioplegia solutions, and produce, among other actions, preservation of mitochondrial function after global ischemia and reperfusion. We have not measured lipid peroxide levels in our ischemic-reperfused hearts to establish definitively the proposed mechanism of action, however.

The major clinical criteria used to assess postischemic cardiac status center on the ability of the heart to contract sufficiently to maintain cardiac output, not on mitochondrial function. This may appear to diminish the importance of our data, which showed improvements only of mitochondrial function. However, postoperative cardiac support frequently involves inotropic drugs which concomitantly increase myocardial oxygen demand. Therefore, although basal postischemic mitochondrial function may be adequate for the hypodynamic heart with its lower oxygen demands, peak (stimulated) oxidative phosphorylating capacity may be inadequate when an inotropic drug is administered, or when adrenergic influences to the heart are increased. Therefore, the ability of dimethyl sulfoxide to maintain mitochondrial function may take on added importance, despite its negligible effect on contractile performance.

We raise the question of whether dimethyl sulfoxide may have more widespread use than it currently has. Posthypoxic or postischemic reoxygen-mediated damage occurs in the heart and brain (22), and it is likely to be a universal phe- 
nomenon, applicable to other organs such as the kidney. Ischemia and hypoxia of varying intensity and duration are common accompaniments of virtually all organ preservation protocols, regardless of whether the organ will be perfused continuously, stored in an ischemic state after an initial flush, or frozen and thawed. We suggest then that since dimethyl sulfoxide can confer some additional protection, does so at concentrations which appear to be innocuous, and are far below those potentially toxic concentrations which are considered to be cryoprotective, further study is warranted, particularly in view of the diverse pharmacological effects of the drug which have heretofore been largely ignored.

\section{SUMMARY}

Isolated perfused rabbit hearts were made globally ischemic for $2 \mathrm{hr}$, then reperfused. For $5 \mathrm{~min}$ before and after ischemia hearts were perfused with hypothermic $\left(20\right.$ or $\left.27^{\circ} \mathrm{C}\right)$, hypoxic, substrate-free cardioplegic solutions, some of which contained $70 \mathrm{~m} M$ dimethyl sulfoxide. Postischemic ventricular pressure development, spontaneous heart rate, coronary flow, lactate dehydrogenase release, tissue $\mathrm{Ca}^{2+}$ content, and in vitro mitochondrial oxidative phosphorylation were used to evaluate the protective effects of the various solutions. Aside from the expected observations that cold cardioplegia lessens ischemic damage, we found that dimethyl sulfoxide gave no indication that it exacerbated ischemic damage or lessened the protection afforded by cardioplegia. We also found that, compared to values measured in comparable drug-free treated hearts, dimethyl sulfoxide significantly improved mitochondrial State 3 respiratory rates, respiratory control, and oxidative phosphorylation rates, and essentially prevented mitochondrial changes due to ischemia and reperfusion. We propose that dimethyl sulfoxide may act as a "scavenger" of cytotoxic free radicals, many of which are known to be generated by mitochondria during reoxygenation. Since hypoxia, ischemia, and reoxygenation are common accompaniments of most organ preservation protocols, we suggest that low concentrations of dimethyl sulfoxide might serve as a useful adjunct to organ preservation in the nonfrozen state, when cryoprotective concentrations are not needed.

\section{REFERENCES}

1. Ashwood-Smith, M. J. Current topics concerning radioprotective and cryoprotective properties of dimethyl sulfoxide in cellular systems. Ann. N.Y. Acad. Sci. 243, 246-256 (1975).

2. Boveris, A., and Chance, B. The mitochondrial generation of hydrogen peroxide. Biochem. $J$. 134, 707-716 (1973).

3. Burges, R. A., Blackburn, K. J., and Spilker, B. Effects of dimethylsulphoxide, dimethyl formamide, and dimethyl acetamide on myocardial contractility and enzyme activity. Life Sci. 8, 1325 - 1335 (1969).

4. Cederbaum, A. I., Dicker, E., Rubin, E., and Cohen, G. The effect of dimethylsulfoxide and other hydroxyl radical scavengers on the oxidation of ethanol by rat liver microsomes. Biochem. Biophys. Res. Commun. 78, 1254-1262 (1977).

5. Conover, T. E. Influence of organic solutes on the reactions of oxidative phosphorylation. J. Biol. Chem. 224, 254-259 (1969).

6. Conover, T. E. Influence of nonionic organic solutes on various reactions of energy conservation and utilization. Ann. N.Y. Acad. Sci. 243, 24-37 (1975).

7. Crapo, J. D., and Tierney, D. F. Superoxide dismutase and pulmonary oxygen toxicity. Amer. J. Physiol. 226, 1401-1407 (1974).

8. Dionisi, O., Galeotti, T., Terranova, T., and Azzi, A. Superoxide radical and hydrogen peroxide formation in mitochondria. Biochim. Biophys. Acta 403, 292-301 (1975).

9. Famaey, J. P., and Whitehouse, M. W. About some biochemical properties of dimethylsulfoxide and three of its homologues: Is the acidic function essential for nonsteroidal antiinflammatory activities? Agents Actions 4, 259-263 (1974).

10. Feuvray, D., and DeLeiris, J. Effect of dimethylsulfoxide on isolated rat heart and lacticodehydrogenase release. Eur. J. Pharmacol. 16, 8-13 (1971).

11. Feuvray, D., and DeLeiris, J. Effect of short di- 
methylsulfoxide perfusions on ultrastructure of the isolated rat heart. J. Mol. Cell. Cardiol. 5, 63-70 (1973).

12. Finney, J. W., Urschel, H. C., Balla, G. A., Race, G. J., Jay, B. E., Pingree, H. P., Dorman, H. L., and Mallams, J. T. Protection of ischemic heart with DMSO alone or DMSO with hydrogen peroxide. Ann N.Y. Acad. Sci. 141, 231-241 (1967).

13. Ganote, C. E., Worstell, J., and Kaltenbach, J. P. Oxygen-induced enzyme release in irreversible myocardial injury: Effects of cyanide in perfused rat hearts. Amer. J. Pathol. 84, 327-343 (1976).

14. Hearse, D. J., Humphrey, S. M., and Bullock, G. R. The oxygen paradox and the calcium paradox: I wo facets of the same problem? $J$. Mol. Cell. Cardiol. 10, 641-668 (1978).

15. Heikkila, R. E. The prevention of alloxan-induced diabetes in mice by dimethyl sulfoxide. Eur.J. Pharmacol. 44, 191-193 (1977).

16. Holtz, G. C., and Davis, R. B. Inhibition of human platelet aggregation by dimethylsulfoxide, dimethylacetamide, and sodium glycerophosphate. Proc. Soc. Exp. Biol. Med. 141, 244-248 (1972).

17. Jynge, P., Hearse, D. J., DeLeiris, J., Feuvray, D., and Braimbridge, M. W. Protection of the ischemic myocardium. Ultrastructural, enzymatic and functional assessment of the efficacy of various cardioplegic infusates. J. Thorac. Cardiovasc. Surg. 76, 2-15 (1978).

18. Kjekshus, J. K., Henry, P. D., and Sobel, B. E. Activation of phosphorylase by cyclic AMP without augmentation of contractility in the perfused guinea pig heart. Circ. Res. 25, 468-478 (1971).

19. Leon, A. S., Bloor, C. M., and Pitt, B. The effects of dimethyl sulfoxide (DMSO) on the healing of experimental myocardial necrosis. Amer. Heart J. 79, 384-389 (1970).

20. Loschen, G., Flohe, L., and Chance, B. Respiratory chain linked $\mathrm{H}_{2} \mathrm{O}_{2}$ production in pigeon heart mitochondria. Fed. Eur. Biochem. Soc. Lett. 18, 261-264 (1971).

21. Loschen, G., Azzi, A., Richter, C., and Flohe, L. Superoxide radicals as precursors of mitochondrial hydrogen peroxide. Fed. Eur. Biochem. Soc. Lett. 42, 68-72 (1974).

22. Majewska, M. D., Strosznajder, J., and Lazarewicz, J. Effect of ischemia, anoxia and barbiturate anesthesia on free radical oxidation of mitochondrial phospholipids. Brain Res. 158, 423-434 (1978).

23. Myers, M. B., and Donovan, W. Effect of dimethyl sulfoxide and hydrogen peroxide on tis- sue gas tensions. Ann. N.Y. Acad. Sci. 243, 320-324 (1975).

24. Panganamala, R. V., Sharma, H. V., Heikkila, R. E., Geer, J. C., and Cornwell, D. G. Role of hydroxyl radical scavengers dimethyl sulfoxide, alcohols and methional in the inhibition of prostaglandin biosynthesis. Prostaglandins 11, 599-607 (1976).

25. Plaa, G. L., and Witschi, H. Chemicals, drugs and lipid peroxidation. Annu. Rev. Pharmacol. 16, 125-141 (1976).

26. Sams, W. H., Jr., Carrol, N. V., and Crantz, P. L. Effects of dimethylsulfoxide on isolatedinnervated skeletal, smooth and cardiac muscle. Proc. Soc. Exp. Biol. Med. 122, 103-107 (1966).

27. Shlafer, M. Pharmacological considerations of cryopreservation. In "Organ Preservation for Transplantation” (A. M. Karow, Jr., and D. E. Pegg, Eds.), pp. 177-212, Dekker, New York, 1981.

28. Shlafer, M., and Karow, A. M., Jr. Ultrastructure-function correlative studies for cardiac cryopreservation. I. Hearts perfused with various concentrations of dimethyl sulfoxide. Cryobiology 8, 280-289 (1971).

29. Shlafer, M., and Karow, A. M., Jr. Pharmacological effects of dimethyl sulfoxide on the mammalian myocardium. Ann. N.Y. Acad. Sci. 243, 110-121 (1975).

30. Shlafer, M., Kane, P. F., and Kirsh, M. M. Superoxide dismutase plus catalase enhance the efficacy of hypothermic cardioplegia to protect the globally-ischemic, reperfused heart. $J$. Thorac. Cardiovasc. Surg. 1982 (In press).

31. Shlafer, M., Matheny, J. L., and Karow, A. M., Jr. Cardiac chronotropic mechanisms of dimethyl sulphoxide: Inhibition of acetylcholinesterase and antagonism of negative chronotrophy by atropine. Arch. Int. Pharmacodyn. Ther. 221, 21-31 (1976).

32. Shlafer, M., Kirsh, M. M., Lucchesi, B. R., Slater, A. D., and Warren, S. Mitochondrial function after global cardiac ischemia and reperfusion: Influences of organelle isolation protocols. Basic Res. Cardiol. 76, 250-266 (1981).

33. Vogel, W. M., and Lucchesi, B. R. An isolated blood-perfused, feline heart preparation for evaluating pharmacological interventions during myocardial ischemia. J. Pharmacol. Methods 4, 291-303 (1980).

34. Wolfe, W. G., and deVries, W. Oxygen toxicity. Annu. Rev. Med. 26, 203-217 (1975).

35. Wroblewski, F., and LaDue, J. S. Lactic dehydrogenase activity in blood. Proc. Soc. Exp. Biol. Med. 90, 210-213 (1955). 